\title{
Wavefront corrective lens for the Subaru Laser Launching Telescope
}

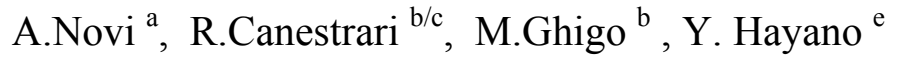 \\ ${ }^{\text {a }}$ Galileo Avionica - via A.Einstein, 35; 50013 Campi Bisenzio - Firenze -Italy \\ ${ }^{\mathrm{b}}$ INAF -Osservatorio Astronomico di Brera - via E.Bianchi, 46; 23807 Merate - Italy(2) \\ ${ }^{\mathrm{c}}$ Università degli Studi dell’Insubria - Via Valleggio 1122100 Como (Co) Italy \\ ${ }^{\mathrm{e}}$ SUBARU Telescope - NAOJ - 650 North A'ohoku Place, Hilo, Hawaii 96720, U.S.A.
}

Email: andrea.novi@selexgalileo.it; rodolfo.canestrari@brera.inaf.it; mauro.ghigo@brera.inaf.it ; hayano@subaru.naoj.org

\begin{abstract}
The optical quality of the LLT unit of SUBARU NAOJ telescope was improved by new athermalized supports of the optics, in order to operate at the best performance at temperatures below $0^{\circ} \mathrm{C}$. The ultimate wavefront correction of the whole LLT, that expands a laser beam from $40 \mathrm{~mm}$ to $500 \mathrm{~mm}$, was made by Ion Beam Figuring on the small 40 $\mathrm{mm}$ LLT entrance window, in accordance to the WFE measured in operating conditions. The correction of small optics including high spatial frequencies, resulting by the LLT expanding ratio, was possible by a special technique of IBF process developed at the Astronomical Observatory of Brera (INAF-OAB), using a concentrator of the ion beam size, able to force the broader beam emitted from an ion source into a smaller spot having large removal rate.
\end{abstract}

Keywords: Laser Launching Telescope, lightweighted mirror, aberration compensating optics, Ion Beam Figuring, WFE correction, removal function, spatial frequencies.

\section{INTRODUCTION}

The Laser Launching Telescope Unit is an afocal system designed to expand, from $40 \mathrm{~mm}$ to $500 \mathrm{~mm}$ diameter, the $589 \mathrm{~nm}$ laser beam of the Laser Guide Star equipment, operating on the NAOJ-SUBARU telescope at Mauna-Kea summit in Hawaii.

The general layout of the LLT Unit is shown in Fig. 1; it is composed by two confocal parabolas, a fold mirror and two borosilicate glass windows (S-BSL7-Ohara), one at entrance and one at exit of the laser beam. The two windows are supported by the Main Structure, that is a box made of light-weighted panels in Aluminium alloy; the primary mirror and the fold mirror, made in Zerodur, are mounted on a baseplate in Aluminium by means of a kinematic mount in Invar. The baseplate also holds the metering structure in CFRP, supporting the secondary mirror.

The "LLT" is the replica of the "LTA" unit [1], originally designed and manufactured for the Laser Guide Star system of the ESO-VLT. Being that assembly to be mounted on top of the VLT telescope secondary mirror, the layout accommodation, in particular the overall maximum allowed height, together with the maximum allowed mass, constituted severe design drivers. These constraints imposed a challenging configuration with fast optics (F number= 1) and forced to lighten either the optics, reducing particularly the primary mirror height, either the mechanical structure. In order to get sufficient thermal-mechanical stability in a short volume, a special kinematic support was designed. Extensive finite elements modelisations were performed to analysis the maintenance of the optical performance ( $60 \mathrm{~nm}$ at full aperture, $55 \mathrm{~nm}$ and $50 \mathrm{~nm}$ respectively at $84 \%$ and $72 \%$ of the aperture) in all different operative conditions, at various attitude positions and operative temperatures. Nevertheless, after integration on the VLT structure, the kinematic mount of the optics did not performed satisfactory at cold temperature as foreseen by the analisys.

The unexpected behaviour was mainly attributed the stiffness difference between the LTA structure in Aluminium and the interface plate to the bay on the ESO-VLT secondary mirror, in combination with the CTE mismatch. Therefore new more sophisticate supports of the mirrors were designed and implemented on the LTA, Fig. 2/b, together with a different mounting flange to the VLT. The upgrade solved the LTA thermal sensitivity.

Advanced Optical and Mechanical Technologies in Telescopes and Instrumentation, edited by Eli Atad-Ettedgui, Dietrich Lemke, Proc. of SPIE Vol. 7018, 701807, (2008) · 0277-786X/08/\$18 · doi: 10.1117/12.787897 
Then the same experienced solution was implemented also on the LLT for Subaru, achieving substantial performance independence respect to temperature changes in the operating range $\left(0^{\circ} \mathrm{C}+/-15^{\circ} \mathrm{C}\right)$. The retrofit was performed directly at SUBARU - Hilo facility. Then the LLT unit was transported at summit where it was aligned and focused at operating conditions. However a residual wavefront error was still found, mainly due to a local deformation like a "volcano" shape in the wavefront near the central hole of M1 where one of the primary mirror new supporting device is bonded (see Fig.2b)

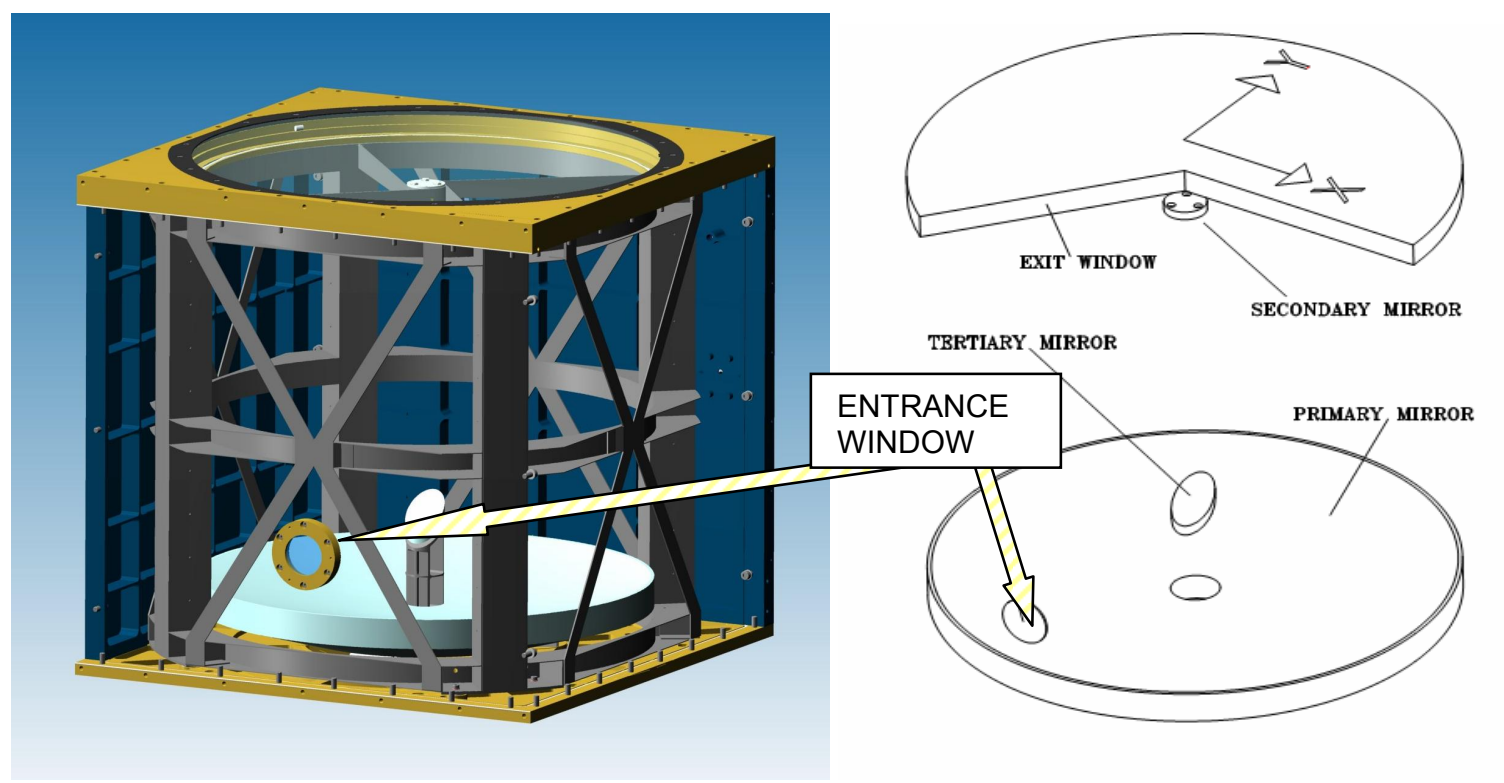

Fig. 1- (a) LLT optical-mechanical configuration ;

(b) Position of the Entrance Window
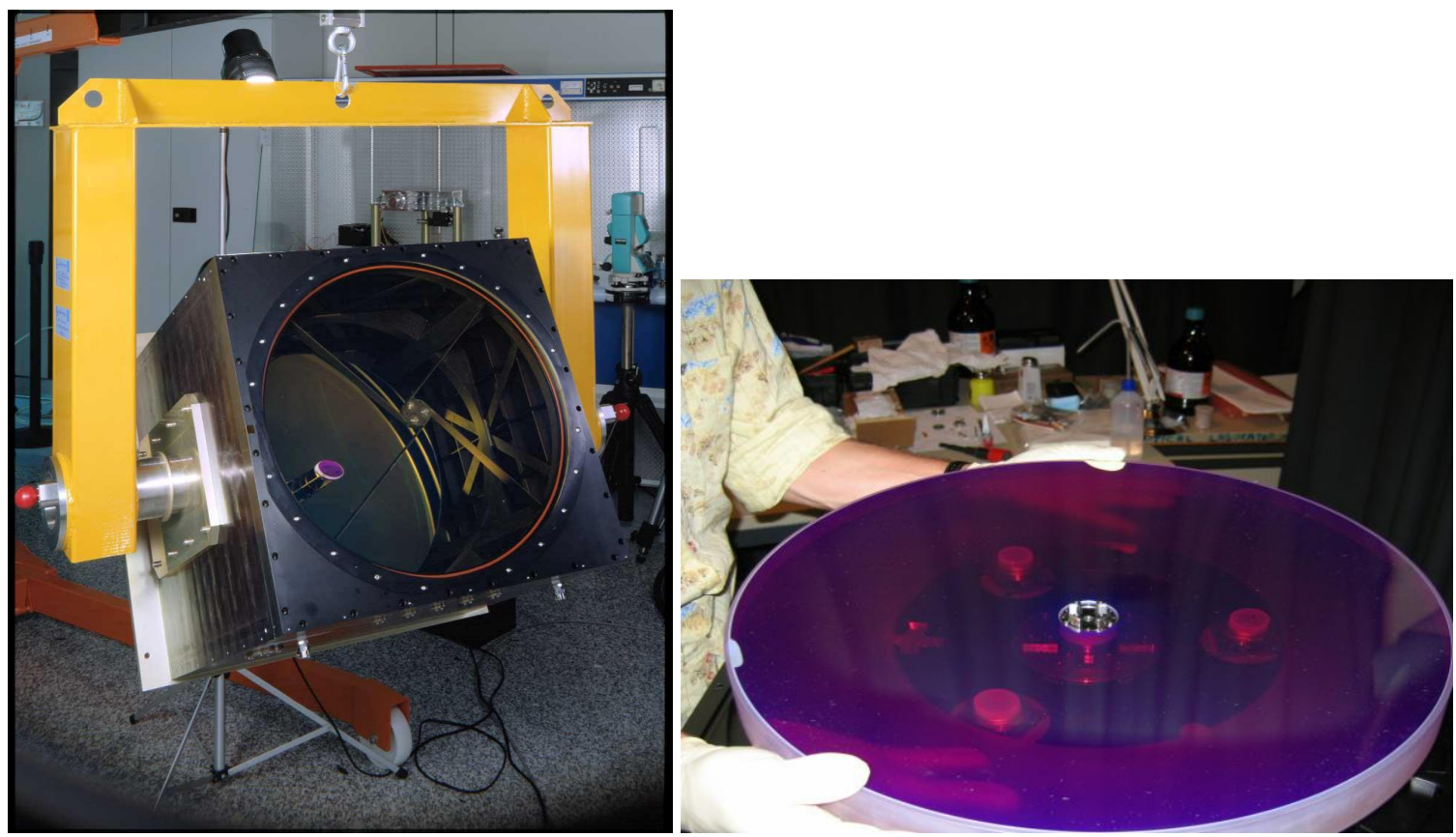

Fig. 2 - (a) The LLT unit ; (b) the Primary Mirror with the new support modified for athermalisation 
This effect was observed at low operating temperature. The cause was attributed to the need of curing the adhesive for $\mathrm{M} 1$ on the central support at $25^{\circ} \mathrm{C}$ : actual the residual $\mathrm{CTE}$ mismatch of Zerodur respect to the Invar causes a small stress at the edge of the support, being the bondig area necessarily near to the optical surface at the centre, being a rather thin mirror. It was sufficient to modify the quality at the central support, giving also small trifoil effect. Aiming to get the best performance at low temperature, an ultimate WFE correction was conceived, to be made on a "compensating" or "nulling" optic, referring to the overall residual WFE, as resulting at low operating temperature and after LLT integration on the telescope.

\section{IBF CORRECTION OF THE LLT ENTRANCE WINDOW}

A possible way for improving the optical performance is to correct one of the optic in the LLT path by Ion Beam Figuring process, creating an "opposite" WFE in one of the optical surfaces, starting from the measured overall WFE. For this purpose the correction of the $500 \mathrm{~mm}$ diameter Exit Windows would be preferable, but it was considered too complex for the aspects of the dismounting and re-coating of the large optic. Therefore the IBF correction was performed by the $40 \mathrm{~mm}$ Entrance Window, which is also easily accessible even on the integrated unit. The error map considered for correction was the one resulting by the LLT once integrated in the SUBARU Telescope at low operating temperature.

The Ion Beam Figuring is a powerful technology to make desired optical corrections, especially when it can be done by removing very low amount of material (in example, below 1 micron). However the correction of WFE on 500 $\mathrm{mm}$ clear aperture become very challenging when "scaled" on the $40 \mathrm{~mm}$ diameter due to the M1/M2 magnification ratio $(12.5 \mathrm{X})$. Actually the spatial frequency of the wavefront errors results scaled also, requiring a very small IBF beam for surface correction. Using small sources, the Gaussian shaped argon ion beam of IBF becomes very weak; a weak IBF beam increases working time and has impact on the accuracy. This limitation of the IBF process was solved using a new IBF working head developed at INAF-OAB in cooperation with Galileo Avionica.

The IBF argon ion source has been equipped with a specially designed (patented) "Ion Concentrator", suitable to make fast removal of material of the surface under correction, starting from an error map with high details or high spatial frequency errors.

In Fig. 3 the concept and the "removal function" are shown, while in Fig. 4 the concept of Ion Concentrator and the relevant hardware. It is based on a well polished cone placed in front of the Argon source grid. This device is equipped with a strong magnetic field produced by rings in Neodymium magnet, placed at the exit of the concentrator, which have a double effect: a) to capture the atoms of metal sputtered from the wall of the cone, avoiding the sputtering on the surface under work; b) acting as a magnetic lens on the Argon ions focusing the beam further. The effectiveness of the IBF technology equipped with this device was already positively tested on a number of small optics [2]. 


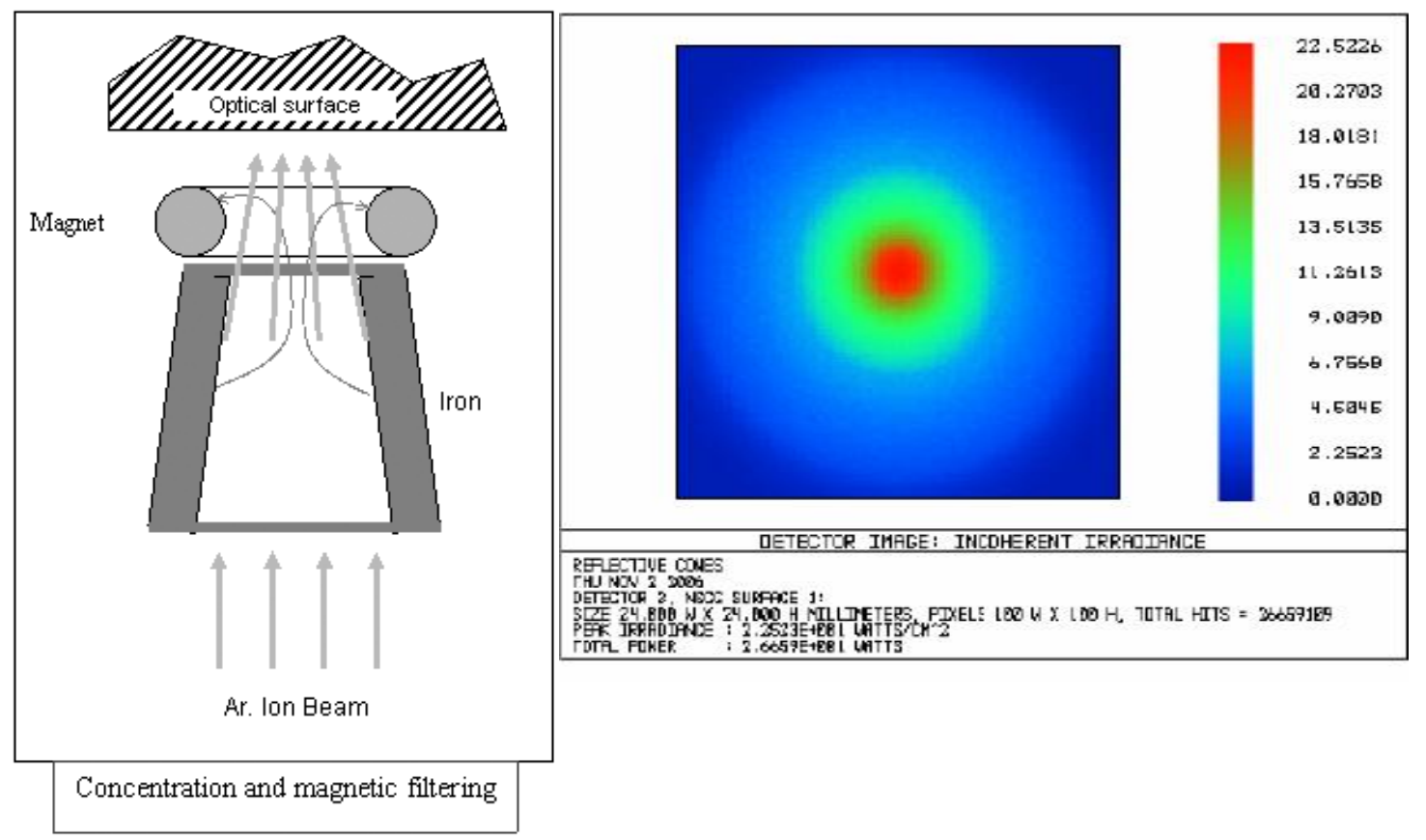

Fig. 3 - (a) IBF with Ionic Concentrator developed at OAB; (b) spot profile, with 6mm (FWHM ) dia. beam

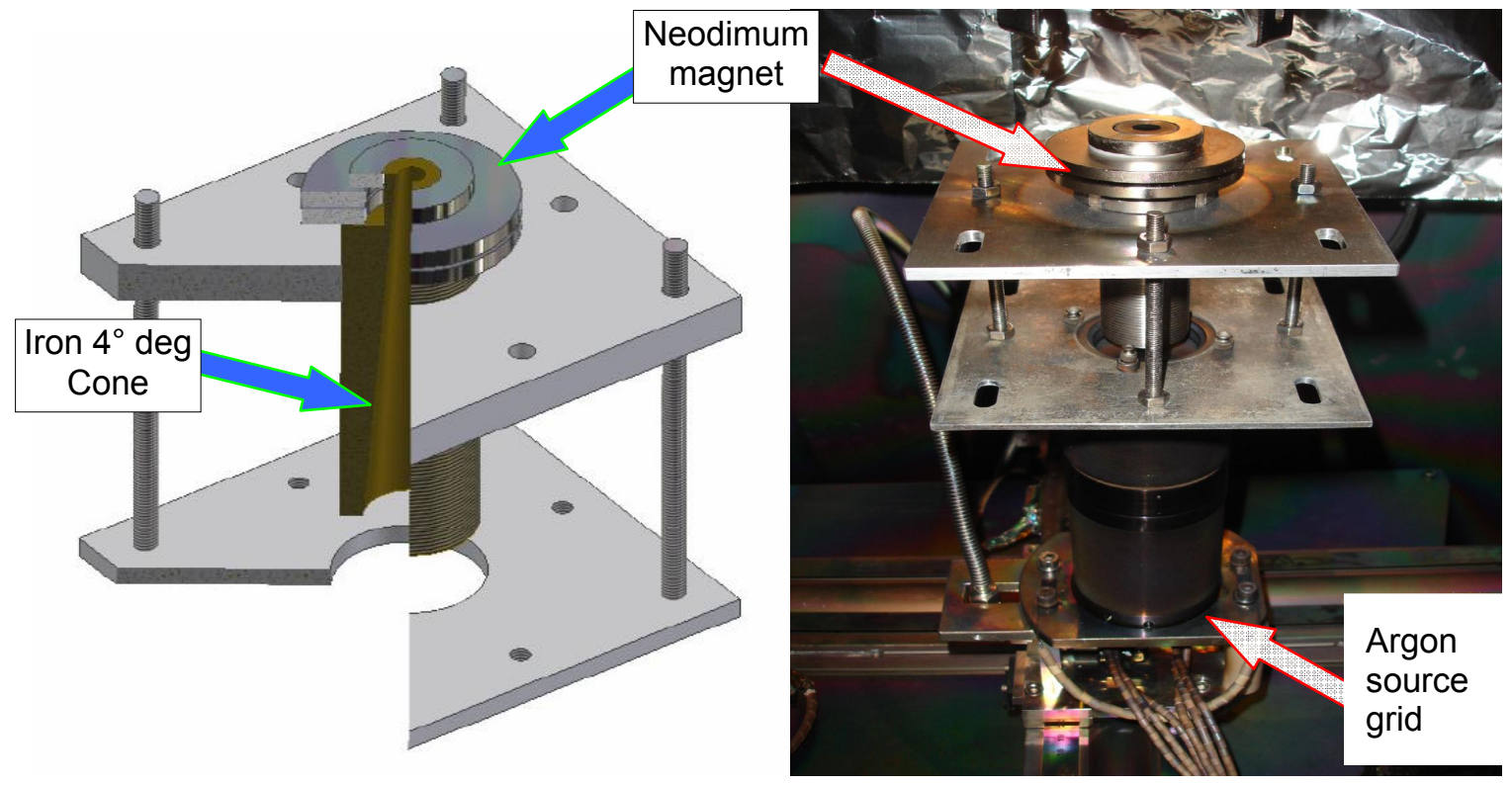

Fig. 4 - Ionic Concentrator of the Ion Beam Figuring at OAB for high spatial frequency correction 


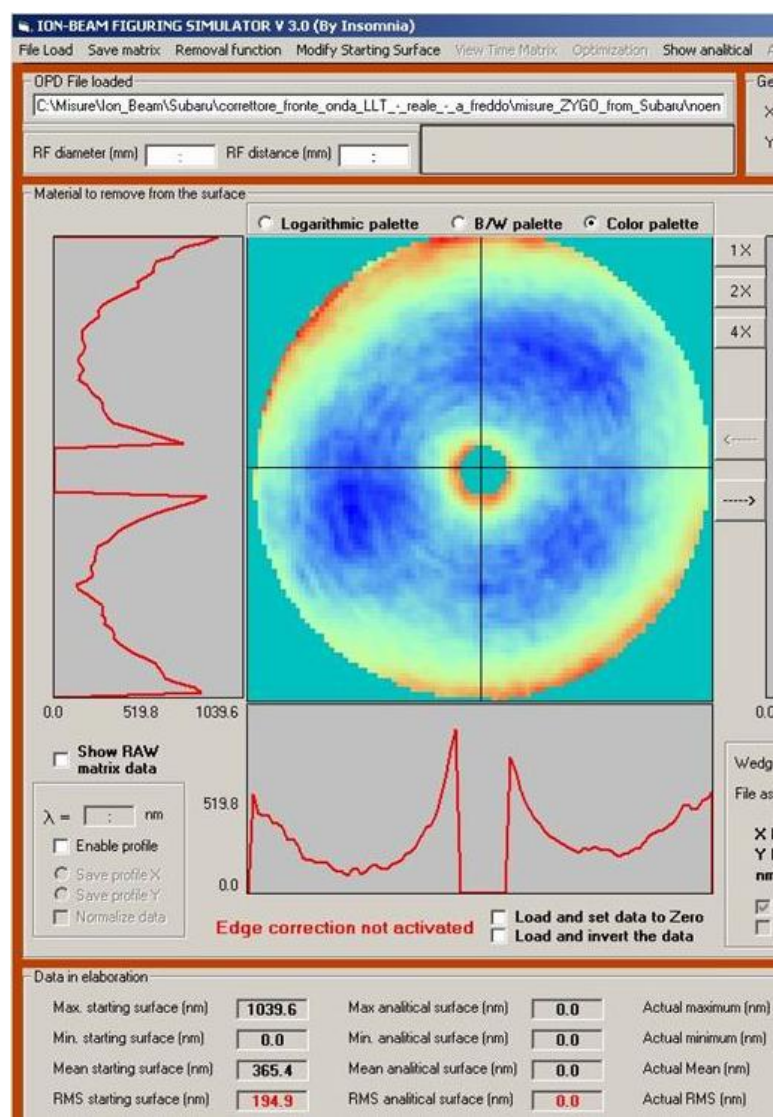

Fig. 5 - (a) Wavefront to be compensated by IBF;

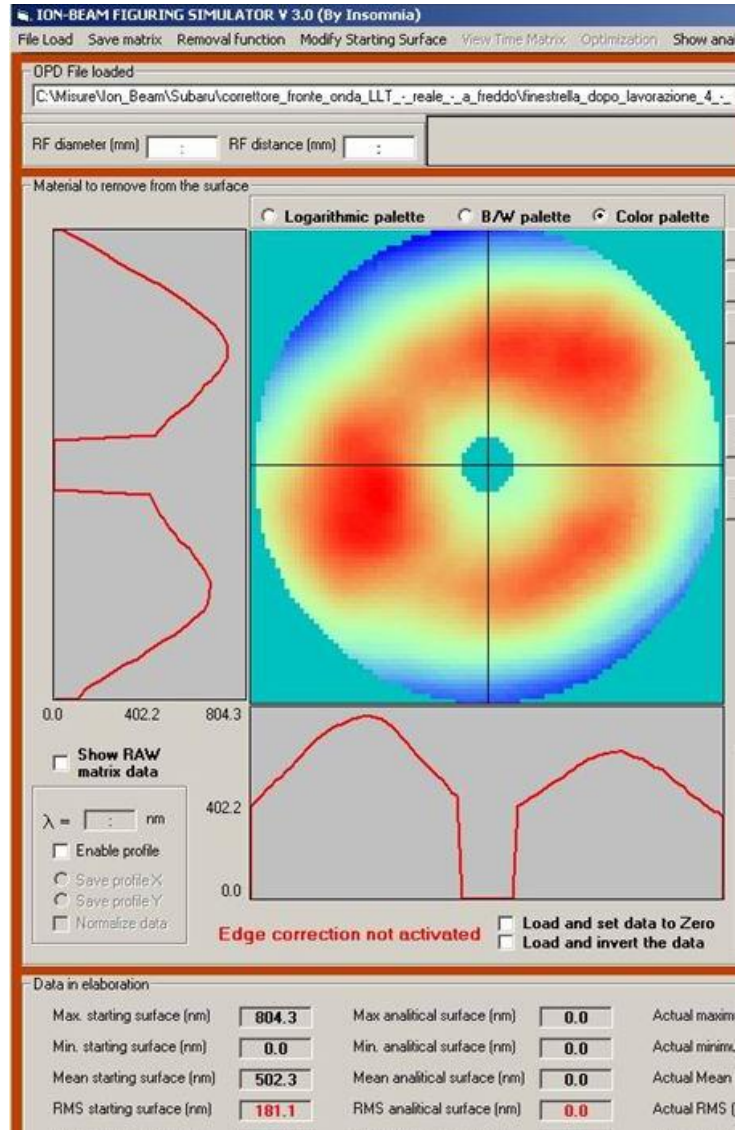

(b) Matrix of the material to be removed on the window.

\section{ANALYSIS OF THE RESULTS}

The error map to be corrected is shown in Fig. 5 (a), being identical to the map of heights that must be "built" on the $40 \mathrm{~mm}$ diameter flat entrance window. To manufacture such profile, the matrix of the material to be removed by IBF is shown in Fig. 5 (b). So the latter map is also proportional to the "dwell-time" or, in other words, the total time of the ion beam remains in a given position of the surface. The map results by the convolution of the removal-function with the WFE map. In our case the removal-function should be computed considering the refraction index of BK7, but this step was not necessary, measuring the removal function directly in transmission on a sample of the same glass material.

Rather, an additional difficulty in shaping the $40 \mathrm{~mm}$ diameter of the LLT is given by the "form" of the error to be corrected in the case in the subject; the mentioned "volcano" peak, has to be "grown" on one surface of the entrance window, just removing the glass material all around it: due to the very small central "scaled" peak, a relatively long working time and iteration of IBF runs were necessary. Moreover the LLT design is characterized by very low central obscuration, therefore the correction has to be made in a very close area of the centre (about 3-4 mm diameter). The not fully compensated area is very small, while the rest of the clear aperture was expected to be flat: according to the simulation of the WFE after insertion of IBF corrected optic, it gave a theoretical result of $51 \mathrm{~nm}$ RMS on the whole $500 \mathrm{~mm}$ diameter LLT clear aperture (Fig. 7/a)

After anti-reflection re-coating, the IBF machined Entrance Window was delivered to NAOJ-SUBARU for integration in the LLT. However the result was not as expected because of a residual astigmatism. The actual result $(77 \mathrm{~nm})$ of the LLT with IBF corrected window is shown in Fig. 6. At the date of preparation of the present paper 
investigations are in progress to verify the cause of the difference (most probably due a trivial error of the handling of input WFE map data for IBF correction). Fig. 7/b is obtained by the actual interferometric results of Fig. 6 after removing by software the astigmatism contribution. Comparing that map, showing WFE $59 \mathrm{~nm}$ RMS, with that of Fig. 7/a we could appreciate the effectiveness of the approach.

Therefore, a new entrance window has been prepared to perform another trial of IBF using the described technology, taking into account the lesson learns of the first trial. At the date of preparation of this paper the work is in progress.

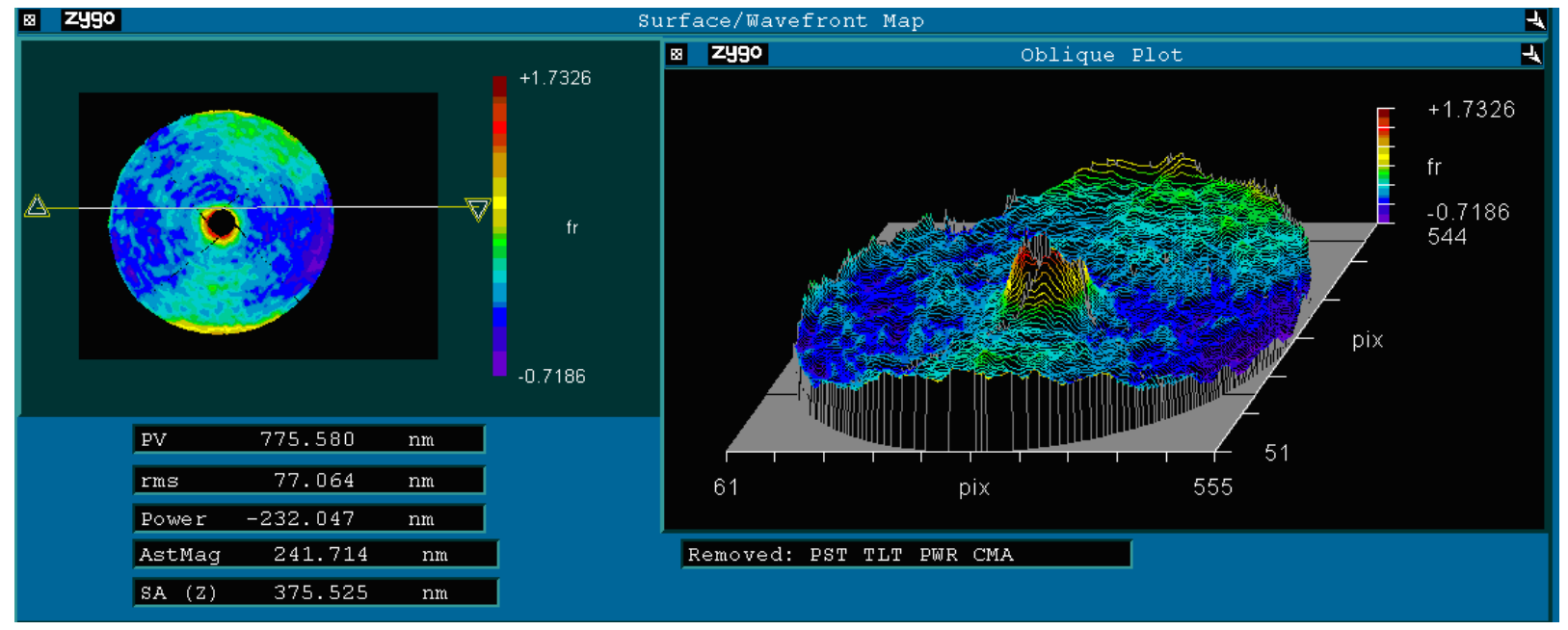

Fig. 6 - First result of with IBF corrected Entrance Window on LLT in operative conditions

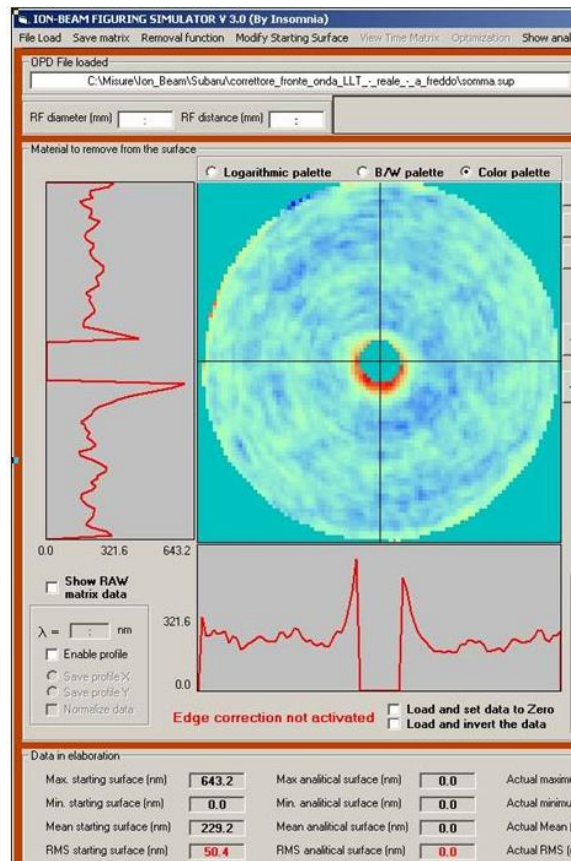

\section{Q Z $\mathbf{Y g O}$}

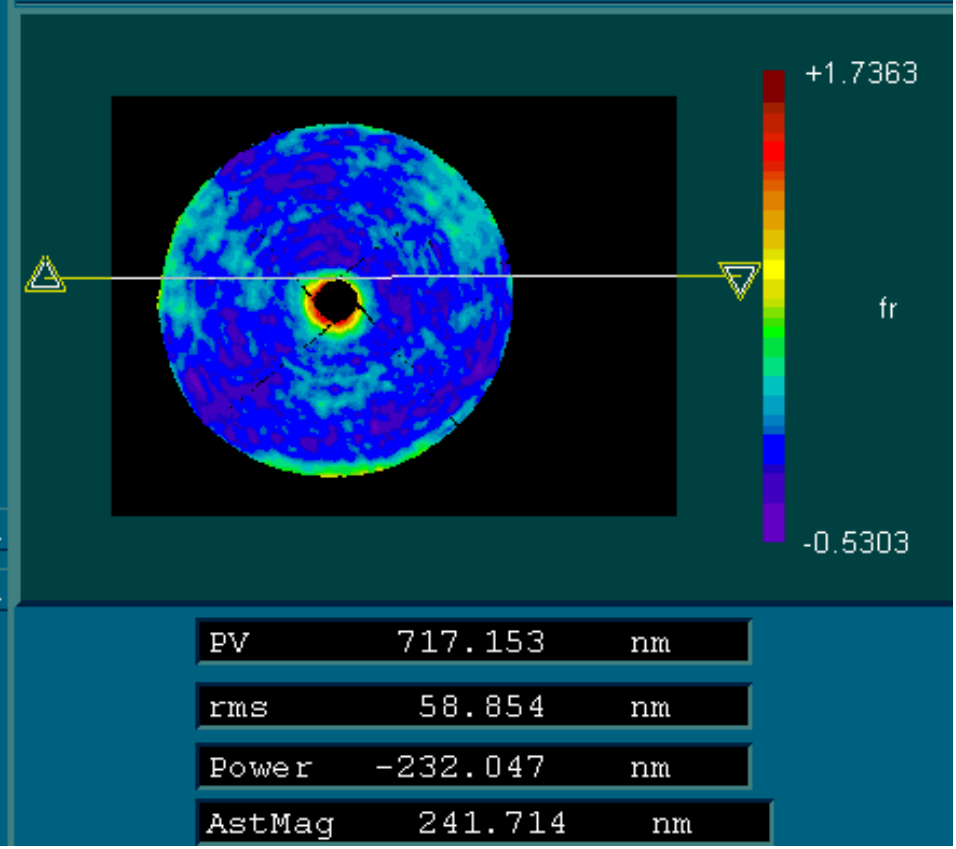

Fig. 7 - (a) Simulation of IBF correction; (b) actual WFE results of LLT with IBF corrected optic (AST subtracted) 


\section{CONCLUSIONS}

A special equipment for Ion Beam Figuring, developed by INAF-OAB in cooperation with Galileo Avionica, was used to make the final WFE correction of the Laser Launching Telescope of the SUBARU-NAOJ, that showed a residual WFE after the change of the primary mirror support. The correction was made on the Entrance Window, that is a $40 \mathrm{~mm}$ diameter optic, taking into account all the final errors of the system once integrated. Due to the magnification factor of the LLT, the correction required to act on high spatial frequency error pattern. A first trial have confirmed the effectiveness of the approach. A new Entrance Window is in progress at the date of the present work; it should give better performance taking into account some lesson learns. The adopted technological solution demonstrated to be powerful and relatively easy; it also has a general validity and it can be proposed for similar cases.

\section{REFERENCES}

[1] Caruso, A., Novi, A. and Basile, G. "Launch Telescope for Astronomical Adaptive optics" SPIE Vol. 5962, 79 (2005).

[2] Ghigo, M., Canestrari, R., Spiga D. and Novi, A. "Correction of high spatial frequency errors on optical surfaces by means of Ion Beam Figuring” SPIE Vol. 6671, 40 (2007). 